\title{
Wachsame Maschinen. Freiräume und Notwendigkeit der Verantwortungsübernahme bei der Entwicklung sozialer Roboter und deren Integration in Bildungsinstitutionen.
}

Ricarda T.D. Reimer und Silvan Flückiger

\begin{abstract}
Dieser Beitrag führt in das Thema "Soziale Robotik in der Bildung" ein und beleuchtet dabei insbesondere medienpädagogisch relevante Dimensionen eines sinnvollen wie kritischen Umgangs mit sozialen humanoiden Robotern. Im Mittelpunkt stehen dabei soziale Roboter als komplexe Phänomene: Sie als Interaktionspartner, Lernbegleiter in Lehrveranstaltungen, aber auch als Projektionsflächen menschlicher Sehnsüchte, Ängste und Zukunftsvorstellungen zu reflektieren, ist Auftrag einer kritisch-reflexiven Medienbildung auf allen Bildungsstufen. Was aber ist ein dem heutigen Erkenntnisstand angemessener und ethisch vertretbarer Einsatz von Robotern in Bildungsinstitutionen? Grundlage dieses Beitrags sind aktuelle Forschungstätigkeiten der Fachstelle Digitales Lehren und Lernen in der Hochschule im Rahmen verschiedener Projekte im Feld „Roboter und Bildung" an der Fachhochschule Nordwestschweiz. ${ }^{1}$
\end{abstract}

\section{Einleitung}

"Social robots have, in the broadest sense, the potential to become part of the educational infrastructure, just as paper, white boards, and computer tablets have" (Belpaeme et al. 2018: 7). Folgt man Belpaemes Annahme, sehen sich Bildungsverantwortliche möglicherweise bald schon vor der Herausforderung, eine technische Innovation in die Lehre zu integrieren, welche in Bezug auf ihr Bildungspotential, die Effizienz der von ihr initi-

1 Einbezogen werden auch damit verbundene Reflexionen in der Fachstelle sowie Ergebnisse der Podiumsdiskussion am Forum Privatheit vom 20.-22. November 2019 in Berlin mit den Beteiligten Isabel Zorn, Ricarda T.D. Reimer und Silvan Flückiger. 
ierten Lernprozesse, den mediendidaktisch sinnvollen Einsatz und den Persönlichkeitsschutz von Lehrenden und Lernenden viele Fragen offenlässt.

Als soziale und humanoide Roboter ${ }^{2}$ interagieren Roboter in direktem Kontakt mit Menschen und nach menschlichem Vorbild. Eine wachsende Zahl an Labor- und Feldstudien weisen den Robotern in Vermittlungsprozessen im weitesten Sinne „pädagogische“ Rollen zu (Belpaeme et al. 2018). Auch wenn ihre technischen Limitationen (noch) offensichtlich $\operatorname{sind}^{3}$, zeigen sich bereits das Lernen unterstützende affektive wie kognitive Effekte (Belpaeme et al. 2018). So sind Roboter als Lernbegleiter beispielsweise in der Lage, die Aufmerksamkeit von Lernenden länger aufrecht zu erhalten. ${ }^{4}$ Um von solchen Effekten profitieren zu können, erfordert der Einsatz eine sorgfältige didaktische Planung und stellt damit Anforderungen an die (medien-)pädagogischen Kompetenzen von Lehrenden. Dies schließt eine Beschäftigung mit ethischen Problemfeldern, welche dem Einsatz sozialer Roboter in Bildungsinstitutionen stets vorgelagert sein sollte, dringend mit ein. In diesem Kontext wird nach den Gestaltungsmöglichkeiten wie auch nach der Verantwortung der Pädagogik bei der Integration sozialer Roboter in Bildungsinstitutionen gefragt. Eine offene und zugleich kritische Auseinandersetzung mit diesen Maschinen erfordert eine vertiefte Beschäftigung - nicht nur mit der technologischen Seite des Phänomens Roboter, sondern auch mit seiner kulturellen, historischen, ökonomischen, gesellschaftlichen oder philosophischen Dimension.

\section{1 (Forschungs-)Gegenstand soziale Roboter}

Soziale Roboter zeichnen sich durch wachsende Autonomie im Bereich Spracherkennung, -verarbeitung und -ausgabe, Bewegung und Entscheidungsfähigkeit aus. Ein humanoides Aussehen und Formen künstlicher In-

2 "Sozial“ heißt in diesem Kontext, dass die Roboter in der Mensch-Roboter-Interaktion Verhalten zeigen, das sich am menschlichen Sozialverhalten orientiert. Im Folgenden werden wir uns auf die Bezeichnung «sozialer Roboter» oder nur «Roboter» beschränken, meinen damit aber stets Roboter, welche in sozialen Interaktionen eingesetzt und humanoid (auch: anthropomorph) gestaltet werden.

3 Yang et al. (2018) liefern eine Übersicht über aktuelle technische Herausforderungen in der Entwicklung sozialer Roboter.

4 Inwiefern diese Effekte auch auf Dauer messbar sind, müssen Längsschnittstudien aufzeigen, welche allerdings noch nicht realisiert sind. 
telligenz verleihen ihnen den Anschein von Kognition und Emotion. Dies ist letztlich der Grund, warum sie für den Einsatz in der realen Lebenswelt der Anwender*innen in Frage kommen. Warum aber werden Roboter für den Einsatz im Klassenzimmer oder an den Hochschulen entwickelt? Welchen pädagogischen Aufgaben, Rollen und Herausforderungen sollen sich diese Maschinen zuwenden?

Auf einer übergeordneten Ebene sieht Michael Decker in den Entwicklungen im Bereich der Sozialrobotik einen Versuch der Verwirklichung eines alten „Menschheitstraums“ (Decker 2010: 46), der darin besteht, den Menschen mit Hilfe der Technik nachzubauen oder ihn gar zu überwinden. Auch Käte Meyer-Drawe deutet die Roboterentwicklungen in ähnlicher Weise: „Die Auseinandersetzung mit der eigenen Vergänglichkeit ist eines der vorherrschenden Motive in der Selbstverfolgung des Menschen mit Hilfe seiner Maschinen“ (Meyer-Drawe 2007: 31). Daneben bietet die Entwicklung humanoider Roboter aber auch Möglichkeiten eines Lernens vom Menschen für den Menschen: Die technische Rekonstruktion menschlicher Organe oder Verhalten ermöglicht sowohl Erkenntnisse zum Beispiel im Bereich der künstlichen Intelligenz als auch ein besseres Verstehen menschlichen Denkens und Handelns (Decker 2010: 47). Die humanoide Gestaltung der Roboter ist weiter als eine Mittel-Zweck-Relation zu deuten: Humanoides Aussehen und Verhalten erleichtern die Interaktion für beide, Mensch und Roboter (z.B. beim Antizipieren von Bewegungen). Roboter sammeln in der Interaktion mit Menschen nützliche Informationen, um sich in deren Verhalten „einzuüben“ (Breazeal 2003). Was ein Vorteil für den Roboter ist, muss nicht zwangsläufig ein Vorteil für den Menschen sein. Wo entstehen Mehrwerte für das lernende Subjekt bei der Entwicklung humanoider Maschinen?

Aktuelle Studien im Bereich der Pädagogik gehen davon aus, dass Roboter zukünftig Lehrkräfte entlasten und neue Lernerlebnisse ermöglichen können (u.a. Belpaeme et al. 2018). Forschung und Entwicklung konzentrieren sich dazu auf den Vor- und Primarschulbereich (Belpaeme et al. 2018). Dabei sind die am häufigsten ausgeführten Aufgaben der Roboter das Vermitteln von Lerninhalten an Gruppen und in 1:1-Settings sowie das Fördern der Aufmerksamkeit und der Motivation. Dementsprechend nehmen Roboter in Lehr-/Lernszenarien unterschiedliche Funktionen ein, die sich aber alle an pädagogischen Rollen orientieren. In der Rolle des Lehrenden, Assistenten oder des Tutors vermitteln Roboter Wissen. Dies können konkrete curriculare Inhalte (Causo et al. 2017) oder Inhalte mit stärkerem Unterhaltungscharakter sein (Kory Westlund et al. 2017). Im Hoch- 
schulprojekt H.E.A.R.T ${ }^{5}$ wird ein Blended-Learning-Format (Flipped Classroom) von einem Roboter in der Rolle des Lebrenden unterstützt (Weber/Zeaiter 2018). In der Rolle des Student-Peers ${ }^{6}$ oder Lernbegleiters steht die Vermittlung von Lerninhalten, das Führen und Motivieren von Lernenden in einem 1:1-Setting im Vordergrund. In der Rolle des Neulings bietet der Roboter den Lernenden die Chance auf einen Rollenwechsel (Lernen durch Lehren), beispielsweise beim Erlernen der Handschrift (Hood et al. 2015). Als „Neuling“ kann der Roboter auch von Grund auf, oder für ein bestimmtes Einsatzszenario, programmiert werden.

\subsection{Lehren und Lernen mit Robotern}

Die humanoide Gestaltung von Aussehen und Verhalten wirken sich auch auf das Lernen selbst aus: Belpaeme et al. (2018) fragen in ihrer Untersuchung von 309 empirischen Einzelstudien nach den Effekten sozialer Roboter auf das Lernen, nach dem Einfluss der Gestaltung und nach den Rollen in pädagogische Kontexten. Dabei stellen sie fest, dass Roboter das Lernen und insbesondere das Erleben der Lernsituation positiv beeinflussen können. Geweckte Neugier oder erhöhte Motivation, sich mit einem Problem zu befassen, kann Verstehen begünstigen, muss es aber nicht: „Furthermore, positive affective outcomes did not imply positive cognitive outcomes, or vice versa." (Belpaeme et al. 2018: 4) In der Metastudie widmen sich die Autor*innen auch dem vielerorts aufgeführten Vergleich zwischen einem physisch anwesenden Roboter und virtuellen Lernhilfen (auch Bots). Hier zeigt sich, dass die Präsenz des Roboters das Gegenüber zu lernförderlichen sozialen Handlungen motiviert (antworten, Fragen stellen etc.). Die Studie zeigt auf, dass mit anwesenden Robotern auch die besseren Lernergebnisse im Vergleich zu virtuellen Lernhilfen erzielt werden können (Belpaeme et al. 2018).

Beim Einsatz als Student-Peer wurde festgestellt, dass die Aufmerksamkeit von Lernenden über längere Zeit aufrechterhalten werden konnte (Belpaeme et al. 2018). Ebenfalls scheint hier ein personalisiertes Verhalten positive Effekte auf das Lernen zu haben (Baxter et al. 2017). Die Effekte in der jeweiligen pädagogischen Interaktion und deren Auswirkungen auf

5 Humanoid Emotional Assistant Robots in Teaching (H.E.A.R.T). Ein Projekt der Universität Marburg unter der Leitung von Prof. Dr. Jürgen Handke.

6 Die Bezeichnung Student-Peer bezieht sich sowohl auf Schüler*innen wie auch auf Studierende. 
die Qualität des Lernens werden durch Faktoren wie das simulierte Geschlecht des Roboters (Reich-Stiebert/Eyssel 2015) oder die Emotionalität der Sprache und Gestik beeinflusst (Kory Westlund et al. 2017). Kennedy, Baxter und Belpaeme (2015) zeigen zudem, dass ein simuliertes, gesteigertes soziales Verhalten des Roboters (z.B. Gestik) für das menschliche Gegenüber auch überfordernd und dadurch nicht mehr lernförderlich sein kann (z.B. weil es die Lernenden ablenkt).

Wie wichtig die Erforschung von (Lern-)Effekten ist, wird vor dem Hintergrund der in der Literatur formulierten Erwartungen an Roboter deutlich (beispielhaft Belpaeme et al. in Bezug auf eine individuelle Betreuung von Lernenden):

„A social robot has the potential to deliver a learning experience tailored to the learner, supporting and challenging students in ways unavailable in current resource-limited educational environments. Robots can free up precious time for human teachers, allowing the teacher to focus on what people still do best: providing a comprehensive, empathic, and rewarding educational experience" (Belpaeme et al. 2018: 7).

Doch können Roboter solchen Zukunftsbildern überhaupt gerecht werden? Welche Herausforderungen und offenen Fragen stehen zwischen aktuellen Robotermodellen und der Vorstellung einer robotergestützten Pädagogik?

\subsection{Offene Fragen und ethische Bedenken}

Die Integration von Robotern in Bildungskontexten wirft angesichts der benannten Einsatzgebiete, vorgesehenen Aufgaben und Rollen eine Reihe ethischer Fragen auf. Humanoid gestaltete und in soziale Interaktionen eingebettete Roboter sind in der Lage, menschliches Verhalten nicht nur zu interpretieren, sondern dieses auch gezielt oder unbeabsichtigt durch ihr Design und ihre technischen Möglichkeiten zu verändern: Siebert et al. (2019) diskutieren in diesem Zusammenhang Roboter, deren Potentiale und Risiken als "persuasive technology». Vollmer et al. (2018) weisen beispielsweise darauf hin, dass mehrere Roboter zusammen Gruppenmeinungen entwickeln, und Kinder so unter Druck setzen könnten. Amanda J. C. Sharkey (2016) entfaltet die folgenden Problemfelder mit Blick auf Roboter im schulischen Unterricht: Bindung (attachment), Täuschung (deception), Verlust menschlicher Kontakte (loss of human contact), Datenschutz (privacy), Kontrolle (control) und Verantwortung (accountability). Die 
Problemfelder, die in Lehr-/Lern-Szenarien besonders zu beachten sind, werden im Folgenden kurz vorgestellt und ergänzt.

Menschen gehen Bindungen mit Mitmenschen ein, können sich aber auch an Objekte wie Maschinen emotional binden. Doch wie wirken sich solche Bindungen auf den Menschen und insbesondere auf Heranwachsende aus?

„[T] his would open the possibility of the children adopting the robot's apparent values, and as in the case of the robot companion, basing their social skills and world outlook on the behaviour and apparent attitudes of a machine rather than on a living, breathing, empathising human" (Sharkey 2016: 291).

Zentrales und aus ethischer Sicht problematisches Element ist dabei die Täuschung. Als Lebendigkeit simulierende Maschinen bedienen sich die programmierten Roboter dem Mittel der Täuschung, um vorgegebene Ziele zu erreichen (z.B. eine vertraute Lernatmosphäre zu gestalten). Sharkey macht im Zusammenhang mit Täuschung zudem darauf aufmerksam, dass von Robotern eine Gefahr ausgehen kann, wenn diese die Illusion wecken, über ein Sein oder Fähigkeiten zu verfügen, die sie in Wirklichkeit nicht haben. Das könnte Menschen dazu verleiten, Roboter für Aufgaben einzusetzen, für die sie nicht geeignet sind. Maschinen, die emotionale Betroffenheit zeigen oder Freundschaften anbieten, vermischen die Kategorien lebendig und maschinenhaft zwar nur scheinbar, sie machen es Menschen jedoch leicht, sich auf das "Spiel“ mit dem scheinbar Lebendigen einzulassen - und sich in diesem Spiel zu verlieren, insbesondere dann, wenn sich der Roboter dem Kind als verständnisvoller Freund inszeniert. Das Kind könnte meinen, der Roboter sei lebendig und es selbst sei dem Roboter tatsächlich wichtig (im Sinne von wertvoll). Irgendwann aber realisiert das Kind, dass die vermeintliche emotionale Nähe bloss ein Algorithmus, das erlebte Verständnis nur eine Illusion war. Welche Auswirkungen hat diese Erfahrung auf künftige Beziehungen? Verständnis, Freundschaft oder Mitgefühl sind nur vorgespielt, nur geschickt programmierte Täuschungen, welchen zumindest potentiell die Gefahr der Ent-Täuschung innewohnt (z.B. durch technische Defekte, fehlende Empathie oder Verfügbarkeit des Geräts), was wiederum das Wohlergehen des Kindes angeht oder sich gar auf andere menschliche Beziehungen auswirkt. ${ }^{7}$ Sharkey spricht von einer „relationship with a psychopath“ (Sharkey 2016: 290) und meint die Tatsa-

7 Der Einwand, Kinder beseelten auch andere Objekte wie Puppen oder Tiere greift deshalb zu kurz, weil Roboter aktiv diese Täuschung unterstützen und die Bezie- 
che, dass wir zwar Empathie und Freundschaft gegenüber einem Roboter empfinden können, dieser aber lediglich so programmiert ist, als würde er diese erwidern, selbst aber keinerlei solcher Gefühle hat. Roboter sind immer nur pseudo-soziale Maschinen (Krotz 2007), welche zwar interagieren können, zu echter Sorge - wie sie in einer pädagogischen Beziehung notwendig wäre - aber nicht in der Lage sind.

Als problematisch beurteilt Sharkey vor allem Roboterrollen wie die des Student-Peer und des Lernbegleiters. Folgerichtig fordert sie eine kritische Folgenabschätzung beim Einsatz sozialer Roboter im Unterricht, vor allem hinsichtlich eines möglichen Verlustes oder der Schädigung menschlicher Kontakte. So könnte das Kind die Gesellschaft eines Roboters der anderer Kinder vorziehen, insbesondere dann, wenn ihm der Roboter stets freundlich gesinnt ist und seine Wertschätzung ihm gegenüber ausdrückt. Die Beziehung zu einem Roboter kann aber nur sehr bedingt als Ersatz für echte menschliche Kontakte dienen.

Brščić et al. (2015) stellen fest, dass bösartiges Verhalten von Kindern gegenüber Robotern meist unbemerkt (wenn das Kind alleine mit dem Roboter ist) und ohne Konsequenzen bleibt. Die Roboterethik diskutiert deshalb darüber, den Roboter als Träger moralischer Werte und Rechte zu definieren (u.a. Loh 2018), nicht nur, um ihn vor Schaden zu bewahren, sondern auch, um den Menschen vor den negativen Auswirkungen seines gewalttätigen Verhaltens gegenüber Robotern zu bewahren (z.B. Empathieverlust in realen menschlichen Beziehungen).

Überdies berühren Roboter noch ein weiteres Problemfeld, nämlich den Schutz von Privatheit bzw. Datenschutz, wobei mindestens fünf Faktoren zentral erscheinen. Erstens ist der Roboter mit Sensoren ausgestattet, die potentiell persönliche und sensible Daten erfassen, verarbeiten und speichern. Die Möglichkeit der Speicherung so erhobener Daten verschärft zweitens die Gefahr von Datenmissbrauch. Wolfert et al. (2020) weisen darauf hin, dass ein Roboter und seine sozialen Fähigkeiten das Ziel von Hacking-Angriffen sein können und so Zugang zu Orten und Informationen offenlegen oder gar zu Handlungen motivieren könnten, welche dem Menschen Schaden zuführen können. Drittens werden Roboter immer autonomer, was ihre Fähigkeit betrifft, auf erhobenen Daten basierende Entscheidungen zu fällen. Viertens werden Roboter mobiler (kleiner, tragbar) was ihnen erlaubt, auch im Privaten, z.B. im Kinderzimmer, eingesetzt zu werden (z.B. als Spielzeug, Nanny oder Aufgabenhilfe). Fünftens offeriert

hung zum menschlichen Gegenüber mitgestalten können, was bei einem anderen Objekt nicht der Fall ist. 
die Gestaltung des Roboters eine neue Art der Nähe zwischen Maschine und Mensch, physisch wie auch emotional. Humanoide Roboter könnten dadurch dem Menschen viel leichter persönliche Informationen entlocken als andere Maschinen, auch, weil die Datenerfassung meist unbemerkt bleibt (z.B. weil man sich unbeobachtet fühlt).

Für den Unterrichtsalltag bedeutet dies, dass ein Roboter fähig sein könnte, einzelne Kinder oder Gruppen von Kindern zu überwachen und deren Verhalten zu analysieren und zu bewerten. Bereits die Verwendung von Technologie zur Ermittlung von Emotionen kann als Verletzung der Privatheit verstanden werden (Sharkey 2016). Außerdem besteht noch keine Einigkeit darüber, wofür die bereits heute verfügbaren Daten verwendet werden könnten und sollten: Wer erhält Zugang zu den Lerndaten der Lernenden? Welche wirtschaftlichen Interessen fließen mit der Datenerhebung in die Unterrichtspraxis mit ein? Welche ethischen Grundsätze sollen berücksichtigt werden? Unklar bleibt ebenfalls, ob und aufgrund welcher Datensätze der Roboter selbständige Entscheidungen in der Interaktion mit Menschen treffen darf. ${ }^{8}$

Wird der Roboter in der Rolle des Lehrenden in einem Klassenverband eingesetzt, stellt sich im Weiteren die Frage nach der Autorität. Roboter in einer entsprechenden Position müssen mit der nötigen Autorität ausgestattet werden, um nicht nur auf geltende Regeln hinzuweisen, sondern diese gegebenenfalls durchzusetzen. Voraussetzung dafür ist, dass der Roboter positives und lernförderliches von negativem und die pädagogische Interaktion störendem Verhalten unterscheiden kann. Ebenso muss er die Vermittlung laufend an die aktuellen Bedürfnisse im Lernraum anpassen können. $\mathrm{Zu}$ beidem sind bisher eingesetzte Robotermodelle (noch) nicht fähig. Es scheint aus heutiger Sicht fraglich, ob Roboter jemals der Komplexität einer pädagogischen Interaktion gerecht werden können. Als Ersatz einer menschlichen Lehrkraft scheidet er deshalb aus: „[R]obots do not have the necessary moral and situational understanding to be able to adequately, or acceptably, fulfil this role.“ (Sharkey 2016: 293)

Entscheidungsmöglichkeiten sozialer Roboter in Lehre oder Erziehung können nicht unabhängig von moralischen Werten diskutiert werden. Die weiterführende Frage lautet dann, wie sich Moral überhaupt in eine Maschine implementieren lässt und ist Teil maschinenethischer Diskurse.

8 Zwar wird die Möglichkeit diskutiert, dass Roboter fairere und unparteiischere Entscheidungen als Menschen treffen könnten, weil sie sich nicht von Emotionen leiten ließen (Sharkey 2016). Allerdings kann der Roboter immer nur so "gut“ sein, wie der Mensch, der ihn programmiert, und nur so objektiv, wie die Daten, auf die er zugreift (Friedman/Nissenbaum 1996). 
Grundsätzlich scheinen drei Ansätze denkbar (Loh 2018: 9f). Moralische (Grund-)Werte könnten in geeignete Programmiersprachen übersetzt und dem Roboter implementiert werden (Top-Down). Abgesehen von der damit verbundenen Übersetzungsherausforderung könnte eine vorab programmierte „Moral“ der Komplexität alltäglicher Entscheidungen nicht gerecht werden (es fehlt an Kontextsensibilität). Die umgekehrte Herangehensweise, diejenige, dass der Roboter nach seinem menschlichen Vorbild Schritt für Schritt lernt, moralische Urteile zu fällen, scheint hier adäquater (Bottom-Up). Da sich ein solches Lernen auf der Basis künstlicher Intelligenz vollziehen würde, scheint die Befürchtung gerechtfertigt, dass der Roboter auch von vermeintlich objektiven Datensätzen und unerwünschten Vorbildern lernen (also bspw. rassistische Urteile fällen oder ganz neue, eigene Maßstäbe entwickeln) könnte. Als Zwischenweg bietet sich darum ein hybrider Ansatz an, bei dem der Roboter gewisse (moralische) Grundsätze implementiert erhält, gleichzeitig aber in begrenztem Ausmaß Autonomie und moralische Sensibilität entwickeln kann (Loh 2018: 10).

Von gesellschaftlicher Seite bedürfte der Einsatz sozialer Roboter in Bildungskontexten der Legitimation, Kontrolle über Menschen auszuüben. Ein Roboter müsste adäquat einschreiten können, wenn Lernende sich selbst oder andere gefährden. Diese Autorität ist aber bisher Erziehungsberechtigten vorbehalten. Daraus ergibt sich der wenig praktische Umstand, dass ein Roboter im Unterricht nie alleine mit Lernenden gelassen werden darf.

Die bisherigen Überlegungen führen zu grundsätzlichen Fragen im Hinblick auf den Einsatz sozialer Roboter im Bildungsbereich. Zentral ist ein Nachdenken über die möglichen pädagogischen Rollen, die Roboter zugewiesen bekommen, sowie ihre Entscheidungsspielräume und -befugnisse hinsichtlich der Lernformen und -inhalte aber auch in der Bewertung der Leistung oder des Verhaltens von Lernenden. „To what extent should robots be trusted to make the right decisions about what humans should do?" (Sharkey 2016: 288) wirft folgerichtig die Frage auf, welchen Platz wir der Robotertechnologie im Bildungswesen zuweisen möchten, oder wie es Belpaeme et al. formulieren: „How far do we want the education of our children to be delegated to machines, and social robots in particular?" (Belpaeme et al. 2018: 7).

\section{Integration in die Lehre und medienpädagogische Implikationen}

Bei der Integration sozialer Roboter in die Lehre sind curriculare Vorgaben und didaktische Ziele, aber auch Aspekte wie Kostenaufwand sowie 
die Medienkompetenzen von Lehrenden und Lernenden zu berücksichtigen. Roboter sinnstiftend in Lehr-/Lernprozesse zu integrieren, erfordert auf Seiten der Lehrpersonen oder einer Assistenz Programmierkenntnisse, welche es erlauben, den Robotereinsatz inhaltlich passend und niveaugerecht zu gestalten sowie auf das jeweilige (medien-)pädagogische Konzept auszurichten, welches den Ansatz der kritisch-reflexiven Medienbildung berücksichtigt. Beobachtungen aus der Praxis zeigen, dass beim derzeitigen technischen Entwicklungsstand ein gewisses „Tüftlertum“ unumgänglich ist. Der Zeitaufwand für die Planung und Umsetzung von robotergestützten Lehr-/Lernszenarien ist dabei, unabhängig des Fachbereichs, beachtlich. Überdies muss der Support der Geräte sichergestellt werden, was wiederum hohe Ansprüche an die Institutionen stellt (IT-Support, Gerätewartung, Transport, Lagerung etc.).

Im Zentrum eines sinnvollen Robotereinsatzes in der Lehre steht aber nicht die Technologie, sondern die (medien-)pädagogischen Implikationen. Grundlage dafür ist die Unterscheidung von Robotern als Lehr-/Lernmittel und Lerngegenstand sowie der Ansatz der kritisch-reflexiven Medienbildung (Reimer 2003, 2019).

\subsection{Bildungspotential sozialer Roboter}

Bisherige Praxiseinsätze konzentrieren sich darauf, den Roboter als Lehrund Lernmittel einzusetzen, vom Spracherwerb bis hin zum Lernen in MINT-Fächern (z.B. Programmieren). Ebenfalls wird explizit sein Unterhaltungswert („Edutainment“) erwähnt. Ein Roboter verfügt allerdings gerade in seiner humanoider Gestalt über ein Bildungspotential, welches über das eines Werkzeuges hinausgeht, nämlich dann, wenn er als Gegenstand reflektiert wird. Den Roboter als Gegenstand zu thematisieren heißt, ihn in seinen aktuellen und künftigen Rollen ernst zu nehmen und ihn als solchen in die Lehre zu integrieren. Dies beinhaltet unter anderem auch seine kulturelle, historische oder ökonomische Dimension, seine Inszenierung als lebendige*r Interaktionspartner*in z.B. in Lehr-/Lernprozessen, wie auch gesellschaftliche oder ethische Fragestellungen.

Im Bereich der Arbeit übernehmen Roboter schon seit Jahrzehnten umfangreiche Aufgaben und drängen nun als soziale Roboter immer stärker auch in nicht-industrielle und soziale Bereiche vor. Hier entlasten sie den Menschen, stellen aber wiederum sein Selbstverständnis im jeweiligen Tätigkeitsbereich in Frage. Diese Veränderungen in den unterschiedlichen Arbeitswelten durch die Robotik müssen im Bereich der betrieblichen Bildung, letztlich aber bereits während der Schul- und Studienzeit reflektiert 
werden. Neben Fragen des Persönlichkeitsschutzes (v.a. von Kindern und Jugendlichen) gilt es auch ökonomische Interessen und damit verbundene Machtstrukturen, die hinter den Entwicklungen der Sozialrobotik stehen, $\mathrm{zu}$ thematisieren.

Die Entwicklungen im Bereich der künstlichen Intelligenz und der Ausdruckskraft der Roboter (Gestik, Mimik, Intonation der Stimme etc.) verringern von außen erkennbare Unterschiede zwischen Maschinen und lebendigen Wesen. Als Lebendigkeit simulierendes Artefakt fordert der Lerngegenstand sozialer Roboter die Lernenden in ihrem Grundverständnis als Mensch, seiner Kognition und Emotionalität heraus. Der Roboter stellt die Frage nach dem Leben selbst, sowie nach dem spezifisch Menschlichen (Brenner 2009).

Erich Fromm (1968: 43f.) machte schon 1968 auf gesellschaftliche Problematiken in Bezug auf die Entwicklung humanoider Roboter aufmerksam:

„The possibility that we can build robots who are like men belongs, if anywhere, to the future. But the present already shows us men who act like robots. When the majority of men are like robots, then indeed there will be no problem in building robots who are like men."

Das Bestreben, Maschinen zu bauen, die „wie wir" sind, sich aber eben doch vom Menschen - durch Unsterblichkeit oder Unfehlbarkeit bspw. unterscheiden, zeugt von einem bestimmten Menschenbild, einer Vision dessen, was der Mensch sein oder werden soll. Fromm beschreibt eine Bewegung beider, Maschine und Mensch, aufeinander zu, ein Ineinandergleiten vermeintlich klar getrennter Entitäten. Humanoide Maschinen als Projektionsflächen menschlicher Sehnsüchte, Ängste und Zukunftsvorstellungen zu reflektieren, ist Auftrag einer kritisch-reflexiven Medienbildung. Eine Konfrontation mit sozialen Robotern in ihrer Mehrdimensionalität birgt immer auch, so die These dieses Beitrags, ein Potential der Irritation und damit der Reflexion eigener Selbst- und Weltverhältnisse. Beispielsweise kann der reichhaltige Korpus dramatischer, prosaischer, zeichnerischer oder filmischer Quellen rund um das Thema Roboter in Lehrveranstaltungen einbezogen werden. Das erlaubt den Lernenden, ihre eigenen (inneren) Bilder von Robotern zu aktivieren und diese zu reflektieren, was ihnen nicht zuletzt ermöglicht, sich in einer dringend nötigen Diskussion um die Entwicklung und Verbreitung dieser Technologie zu positionieren. 


\subsection{Roboter in Bildungsinstitutionen: Mitgestalten, Mitverantworten}

Der Roboter als Kulturerzeugnis, seine Rolle in einer vom Digitalen geprägten Welt, seine technologische, historische und symbolische Dimension - all dies sind Facetten des Phänomens Roboter. Die Integration von Robotern in die Lehre bedingt, diese Perspektiven stets mitzudenken, sie curricular und fächerübergreifend im Schul- und Hochschulalltag zu verankern. Medienbildung heißt, die Frage nach der gesellschaftlichen, kulturellen und ethischen Dimension technischer Innovationen und ihren Folgen ins Zentrum zu rücken: "Sowohl in der Schule als auch an den Universitäten darf der vermehrte Einsatz der neuen Medien ${ }^{9}$ - als Unterrichtsmittel und Forschungsinstrument - nicht das Reden über sie verdrängen“ (Simanowski 2018: 23). Für einen kritischen Umgang und eine reflektierte Anwendung digitaler Technologien erweist sich die kritisch-reflexive Medienbildung als ein Ansatz für die Lehre, der basierend auf einem klassischen Bildungsbegriff, Urteilsvermögen, Kreativität und Kritikfähigkeit betont und Bildung nicht auf Arbeitsmarktbefähigung reduziert. Ziel ist ein mündiger Umgang mit Technologie und mit den eigenen Vorstellungen und (Zukunfts-)Phantasien davon.

Auf der Ebene der Institution bedeutet dies, sich der Herausforderung sozialer Roboter zu stellen und die eigene Verantwortung wahrzunehmen. Dass Roboter sich in der Lehre etablieren werden, wird in der Forschung kaum in Frage gestellt. Es scheint allein eine Frage der technischen Innovation und damit der Zeit, bis Roboter routinemäßig in Bildungsinstitutionen zum Einsatz kommen. Eine solche Formulierung negiert allerdings den Gestaltungsfreiraum und die daraus abzuleitende Verantwortung der Institutionen bei der Integration von Robotern. Eine kritisch-reflexive Medienbildung weist auf diesen Freiraum sowie die Rolle und Verantwortung der Pädagogik in dieser Entwicklung hin. Letztendlich müssen es Pädagog*innen selbst sein, die über Form und Umfang von Robotereinsätzen in Bildungsinstitutionen entscheiden, begründet auf theoretischen und empirischen Grundlagen sowie medienpädagogischen, insbesondere mediendidaktischen Überlegungen.

Diese Mitgestaltung sollte bereits im Stadium der Forschung und der Entwicklung von Robotertechnologien für den Bildungsbereich erfolgen, indem pädagogische Expertise in Forschungsprojekten zwingend eingefor-

9 Wenngleich Simanowski hier Roboter nicht explizit als neue Medien nennt, kann seine Aussage in diesem Sinne interpretiert werden und in die Argumentation miteinfliessen. 
dert wird. Hier gilt es, pädagogische Fragen im Zusammenhang mit Robotern über interdisziplinär breit abgestützte Forschungsprojekte zu bearbeiten und weiterzuentwickeln. Dabei sind Forscher"innen auch auf entsprechende Mittel angewiesen, was Bildungspolitik und Hochschulleitungen in die Verantwortung miteinbezieht. Die Entwicklung soll nicht allein privatwirtschaftlichen Interessen überlassen werden. Die im Rahmen des Forschungsprojekts FHNW Robo-Lab durchgeführten Workshops sind nur eine Möglichkeit, Teilbereiche der kritisch-reflexiven Medienbildung mit Studierenden und weiteren Bildungsverantwortlichen in Bezug auf soziale Roboter zu stärken (Flückiger/Reimer 2021). Konkret können Lernende bspw. die Aufgabe erhalten, einen idealen Roboter zu entwerfen, und zwar im Hinblick auf dessen Funktionen, Rollen, Aufgaben und moralische Handlungsautonomie. Erfahrungen im Projekt zeigen, dass Studierende den Robotereinsatz als außergewöhnliches Erlebnis im Studierendenalltag schätzen. Gleichzeitig fällt auf, dass die ethische und gesellschaftliche Relevanz sozialer Roboter unterschätzt wird. Eine Diskussion der unterschiedlichen Dimensionen eines Roboters muss aktiv angeregt werden, sonst findet sie nicht statt.

\section{Diskussion}

Was folgt aus den voranstehenden Überlegungen hinsichtlich der Integration sozialer Roboter in die Lehre für die einzelnen Bildungsinstitutionen? Roboter können einerseits bestehende pädagogische Praktiken übernehmen oder ergänzen, indem sie zum Beispiel Wissen personalisiert vermitteln. Andererseits sollen sie neue Unterrichtspraxen ermöglichen, die es den Lehrkräften erlauben, sich noch stärker ihren Kernkompetenzen im direkten Umgang mit Lernenden zuzuwenden. Die Erwartungen an soziale Roboter sind aber hoch und auch dann, wenn man dem verbreiteten Optimismus im Kern zustimmt, bleibt zweifelhaft, ob diese Erwartungen realistisch sind. Dies liegt einerseits an technischen Herausforderungen, welche die pädagogische Praxis an die Roboter stellt. Andererseits bleiben eine Reihe ethischer Fragen offen, welche sich unter anderem aus der humanoiden Gestaltung der Geräte in Verbindung mit einer vulnerablen Zielgruppe (z.B. Kinder, Menschen mit Beeinträchtigungen) ergeben. Die humanoide Gestaltung von Mensch-Maschinen-Interaktionen sollte daher besondere Aufmerksamkeit erhalten. Einer gezielt simulierten Vermischung von Maschinen mit genuin menschlichen Fähigkeiten wie Emotionalität, Empathie, Bindungsfähigkeit, Vertrauen oder Freundschaft - und damit einer Beförderung einer Täuschung - ist kritisch zu begegnen. Gera- 
de die Pädagogik muss dem würdevollen und achtsamen Umgang mit dem Menschen und seiner Privatheit in der Interaktion stets von Neuem einen zentralen Platz einräumen. Grundsätzlich ist es angezeigt, der Einführung sozialer Roboter in unterschiedliche Bereiche der Bildung die Fragen vorwegzustellen, welche konkreten Probleme soziale Roboter im Bildungssystem lösen können, welche sie verschärfen und welche neuen Probleme sie schaffen könnten. Der Einsatz sozialer Roboter in Vor- und Primarschuleinrichtungen ist nach dem heutigen Kenntnisstand nur unter Begleitung medienpädagogisch geschulter Pädagogen und Pädagoginnen und mit klaren didaktischen Zielsetzungen gerechtfertigt. Ebenfalls muss auf allen Bildungsstufen über eine adäquate, d.h., kritisch-reflexive Form der Medienbildung nachgedacht werden, welche den Roboter in seiner Ganzheit erfasst und ihn als Gegenstand zu thematisieren versteht.

\section{Literatur}

Baxter, Paul / Ashurst, Emily / Read, Robin / Kennedy, James / Belpaeme, Tony (2017): Robot Education Peers in a Situated Primary School Study: Personalisation Promotes Child Learning. In: PloS One 12(5):e0178126.

Belpaeme, Tony / Kennedy, James / Ramachandran, Aditi / Scassellati, Brian / Tanaka, Fumihide (2018): Social Robots for Education: A Review. In: Science Robotics 3 (21).

Breazeal, Cynthia (2003): Toward Sociable Robots. In: Robotics and Autonomous Systems 42 (3-4), S. 167-175.

Brenner, Andreas (2009): Leben. Stuttgart: Philipp Reclam jun.

Brščić, Drazen / Kidokoro, Hiroyuki / Suehiro, Yoshitaka / Kanda, Takayuki (2015): Escaping from Children's Abuse of Social Robots. In: Proceedings of the Tenth Annual ACM/IEEE International Conference on Human-Robot Interaction - HRI '15. Portland, Oregon, USA: ACM Press, S. 59-66.

Causo, Albert / Zin Win, Phyo / Peng Guo, Sheng / Chen, I-Ming (2017): Deploying social robots as teaching aid in pre-school K2 classes: A proof-of-concept study. In: 2017 IEEE International Conference on Robotics and Automation (ICRA), S. 42644269.

Decker, Michael (2010): Ein Abbild des Menschen: Humanoide Roboter. In: Information und Menschenbild 37 (1). Berlin, Heidelberg: Springer, S. 41-62.

Flückiger, Silvan / Reimer, Ricarda T.D. (2021) (im Erscheinen): Projektbericht FHNW Robo-Lab-Schwerpunkt „Bildung und Roboter".

Friedman, Batya / Nissenbaum, Helen (1996): Bias in computer systems. In: ACM Transactions on Information Systems 14(3), S. 330-347.

Fromm, Erich (1968): The revolution of hope: toward a humanized technology. New York: HarperCollins. 
Hood, Deanna / Lemaignan, Séverin / Dillenbourg, Pierre (2015): When Children Teach a Robot to Write: An Autonomous Teachable Humanoid Which Uses Simulated Handwriting. In: 10th ACM/IEEE International Conference on Human-Robot Interaction (HRI), S. 83-90.

Yang, Guang-Zhong / Bellingham, Jim / Dupont, Pierre E. / Fischer, Peer / Florid, Luciano / Full, Robert / Jacobstein, Neil / Kumar, Vijay / McNutt, Marcia / Merrifield, Robert / Nelson, Bradley J. / Scassellati, Brian / Taddeo, Mariarosaria / Taylor, Russell / Veloso, Manuela / Lin Wang, Zhong / Wood, Robert (2018): The Grand Challenges of Science Robotics. In: Science Robotics 3(14), S. 1-14.

Kennedy, James / Baxter, Paul / Belpaeme, Tony (2015): The Robot Who Tried Too Hard: Social Behaviour of a Robot Tutor Can Negatively Affect Child Learning. In: 10th ACM/IEEE International Conference on Human-Robot Interaction (HRI), S. 67-74.

Kory Westlund, Jacqueline M. / Jeong, Sooyeon / Park, Hae W. / Ronfard, Samuel / Aradhana, Adhikari / Harris, Paul L. / DeSteno, David / Breazeal, Cynthia L. (2017): Flat vs. Expressive Storytelling: Young Children's Learning and Retention of a Social Robot's Narrative. In: Frontiers in Human Neuroscience 11(295), S. 1-20.

Loh, Janina (2018): Maschinenethik und Roboterethik. In: Oliver Bendel (Hg.): Handbuch Maschinenethik. Wiesbaden: Springer Fachmedien, S. 1-19.

Meyer-Drawe, Käte (2007): Menschen im Spiegel ihrer Maschinen. München: Wilhelm Fink Verlag.

Reich-Stiebert, Natalia / Eyssel, Friederike (2015): Learning with Educational Companion Robots? Toward Attitudes on Education Robots, Predictors of Attitudes, and Application Potentials for Education Robots. In: International Journal of Social Robotics 7(5), S. 875-888.

Reimer, Ricarda T.D. (2003): Medienpädagogische Gestaltungsideen zur Integration von E-Learning in der Hochschullehre. In: Online-Zeitschrift MedienPädagogik. Online verfügbar unter: http://www.medienpaed.com/03-1/reimer03-1.pdf (Abfrage am: 22.04.2020).

Reimer, Ricarda T.D. (2019): Bildungsverantwortung der Hochschulen im Zeitalter der Digitalisierung. In: Marlene Miglbauer / Lene Kieberl / Stefan Schmid (Hg.): Hochschule digital.innovativ I \#digiPH. Tagungsband zur 1. Online-Tagung. Norderstedt: Books on Demand GmbH, S. 23-34.

Siebert, Scarlet / Tolksdorf, Nils / Rohlfing, Katharina / Zorn, Isabel (2019): Raising Robotic Natives?: Persuasive Potentials of Social Robots in Early Education. In: The Journal of Communication and Media Studies 4(4), S. 21-35.

Sharkey, Amanda J. C. (2016): Should We Welcome Robot Teachers? In: Ethics and Information Technology 18(4), S. 283-297.

Simanowski, Roberto (2018): Stumme Medien. Vom Verschwinden der Computer in Bildung und Gesellschaft. Berlin: Matthes \& Seitz.

Vollmer, Anna-Lisa / Mühlig, Manuel / Steil, Jochen J. / Pitsch, Karola / Fritsch, Jannik / Rohlfing, Katharina J. / Wrede, Britta (2014): Robots Show Us How to Teach Them: Feedback from Robots Shapes Tutoring Behavior during Action Learning. In: PLoS ONE 9(3): e91349. https://doi.org/10.1371/journal.pone.0091349. 
Weber, Katharina / Zeaiter, Sabrina (2018): Project H.E.A.R.T. (Humanoid Emotional Assistant Robots in Teaching). In: J. Buchner / Chr. Freisleben-Teutscher / J. Haag \& E. Rauscher (Hg.): Inverted Classroom. Vielfältiges Lernen. Begleitband zur 7. Konferenz Inverted Classroom and Beyond 2018; FH St. Pölten, 20. \& 21. Februar 2018, Brunn am Gebirge: ikon Verlag, S. 237-244.

Wolfert, Pieter / Deschuyteneer, Jorre / Oetringer, Djamari / Robinson, Nicole / Belpaeme, Tony (2020): Security Risks of Social Robots Used to Persuade and Manipulate: A Proof of Concept Study. In: Companion of the 2020 ACM/IEEE International Conference on Human-Robot Interaction. Cambridge United Kingdom: ACM, S. 523-525. 